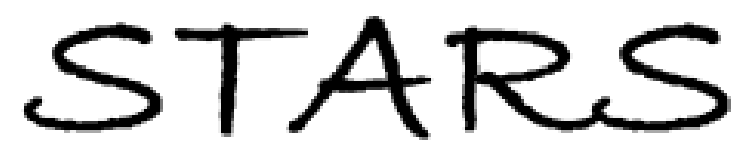

University of Central Florida

STARS

$1-1-2010$

\title{
Effective elastic properties of a van der Waals molecular monolayer at a metal surface
}

Dezheng Sun

Dae-Ho Kim

Duy Le

University of Central Florida

Øyvind Borck

Kristian Berland

See next page for additional authors

Find similar works at: https://stars.library.ucf.edu/facultybib2010

University of Central Florida Libraries http://library.ucf.edu

This Article is brought to you for free and open access by the Faculty Bibliography at STARS. It has been accepted for inclusion in Faculty Bibliography 2010 s by an authorized administrator of STARS. For more information, please contact STARS@ucf.edu.

\section{Recommended Citation}

Sun, Dezheng; Kim, Dae-Ho; Le, Duy; Borck, Øyvind; Berland, Kristian; Kim, Kwangmoo; Lu, Wenhao; Zhu, Yeming; Luo, Miaomiao; Wyrick, Jonathan; Cheng, Zhihai; Einstein, T. L.; Rahman, Talat S.; Hyldgaard, Per; and Bartels, Ludwig, "Effective elastic properties of a van der Waals molecular monolayer at a metal surface" (2010). Faculty Bibliography 2010s. 845.

https://stars.library.ucf.edu/facultybib2010/845

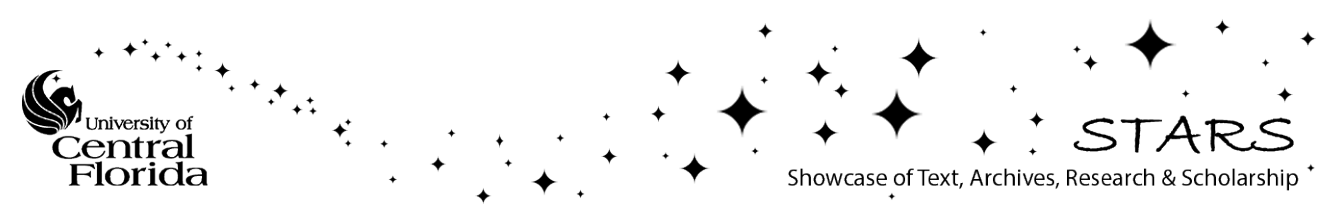




\section{Authors}

Dezheng Sun, Dae-Ho Kim, Duy Le, Øyvind Borck, Kristian Berland, Kwangmoo Kim, Wenhao Lu, Yeming Zhu, Miaomiao Luo, Jonathan Wyrick, Zhihai Cheng, T. L. Einstein, Talat S. Rahman, Per Hyldgaard, and Ludwig Bartels 


\title{
Effective elastic properties of a van der Waals molecular monolayer at a metal surface
}

\author{
Dezheng Sun, ${ }^{1}$ Dae-Ho Kim, ${ }^{1}$ Duy Le, ${ }^{2}$ Øyvind Borck, ${ }^{3}$ Kristian Berland, ${ }^{4}$ Kwangmoo Kim, ${ }^{5}$ Wenhao Lu, ${ }^{1}$ Yeming Zhu, ${ }^{1}$ \\ Miaomiao Luo, ${ }^{1}$ Jonathan Wyrick, ${ }^{1}$ Zhihai Cheng, ${ }^{1}$ T. L. Einstein, ${ }^{5}$ Talat S. Rahman, ${ }^{2}$ Per Hyldgaard, ${ }^{4}$ and \\ Ludwig Bartels ${ }^{1, *}$ \\ ${ }^{1}$ Departments of Chemistry and Physics, University of California at Riverside, Riverside, California 92521, USA \\ ${ }^{2}$ Department of Physics, University of Central Florida, Orlando, Florida 32816, USA \\ ${ }^{3}$ Department of Physics, Norwegian University of Science and Technology, Hogskoleringen 5, NO-7491 Trondheim, Norway \\ ${ }^{4}$ Department of Microtechnology and Nanoscience, MC2, Chalmers University of Technology, SE-41296 Göteborg, Sweden \\ ${ }^{5}$ Department of Physics, University of Maryland, College Park, Maryland 20742-4111, USA
}

(Received 25 October 2010; published 23 November 2010; publisher error corrected 23 November 2010)

\begin{abstract}
Adsorbing anthracene on a $\mathrm{Cu}(111)$ surface results in a wide range of complex and intriguing superstructures spanning a coverage range from 1 per 17 to 1 per 15 substrate atoms. In accompanying first-principles density-functional theory calculations we show the essential role of van der Waals interactions in estimating the variation in anthracene adsorption energy and height across the sample. We can thereby evaluate the compression of the anthracene film in terms of continuum elastic properties, which results in an effective Young's modulus of $1.5 \mathrm{GPa}$ and a Poisson ratio $\approx 0.1$. These values suggest interpretation of the molecular monolayer as a porous material—in marked congruence with our microscopic observations.
\end{abstract}

DOI: 10.1103/PhysRevB.82.201410 PACS number(s): 68.60.Bs, 07.79.Fc, 62.20.D-, 71.15.Mb

Understanding how materials respond to stress and, subsequently, having the ability to predict the resultant deformation (strain) are fundamental to virtually all practical applications of materials. In-depth understanding of materialspecific stress-strain relationships impacts a wide range of scientific fields as varied as the geological deformation of rock $^{1}$ during plate tectonics and the posture-induced deformation of lumbar disks resulting in herniation. ${ }^{2}$ It also has significance for applications at the nanoscale: e.g., in the development of molecular-scale coatings, knowledge of the elastic properties of the employed materials is essential for predicting their ability to maintain integrity under the deformation of their substrate. Yet investigations of molecular overlayers at solid surfaces have generally not addressed issues related to the elastic properties of films, with a few exceptions such as cantilever measurements of magnetostriction. ${ }^{3-5}$ Currently the formation of molecular networks at metal surfaces garners significant attention because of the patterns of unprecedented size and versatility that are produced. ${ }^{6-10}$ The present study aims to complement the advances in creating molecular surface patterns by developing a framework for understanding their elastic properties.

The focus of this study is a system that was chosen because of the simplicity of the geometry of its components and of their interaction with one another: anthracene on $\mathrm{Cu}(111)$. Anthracene consists of three fused benzene rings (Fig. 1 inset) and adsorbs flat on $\mathrm{Cu}(111)$, similar to other acenes. ${ }^{11-17}$ Dispersive or van der Waals (vdW) interactions play an important role in adsorption of acenes (such as benzene) on low-corrugation metal surfaces. In a prior study ${ }^{18}$ some of us addressed the physisorption of benzene and found that with density-functional theory (DFT) calculations in the generalized gradient approximation, a negligible adsorption energy of $\sim 20 \mathrm{meV}$ results, ${ }^{19}$ which favors the ring centered over a hollow site compared to an atop site by $\sim 7 \mathrm{meV}$. In contrast, in similar calculations that also account for $\mathrm{vdW}$ interactions, ${ }^{20}$ markedly higher adsorption energies of $0.55 \mathrm{eV}$ and $0.53 \mathrm{eV}$ at hollow and atop sites, respectively, are found. We extend such calculations to anthracene on $\mathrm{Cu}(111)$ and find substantially higher binding energies of $0.95 \mathrm{eV}$ and $0.93 \mathrm{eV}$ at hollow and bridge sites, respectively, [see Figs. $1(\mathrm{a})$ and $1(\mathrm{~b})]$. There is a similar difference in adsorption
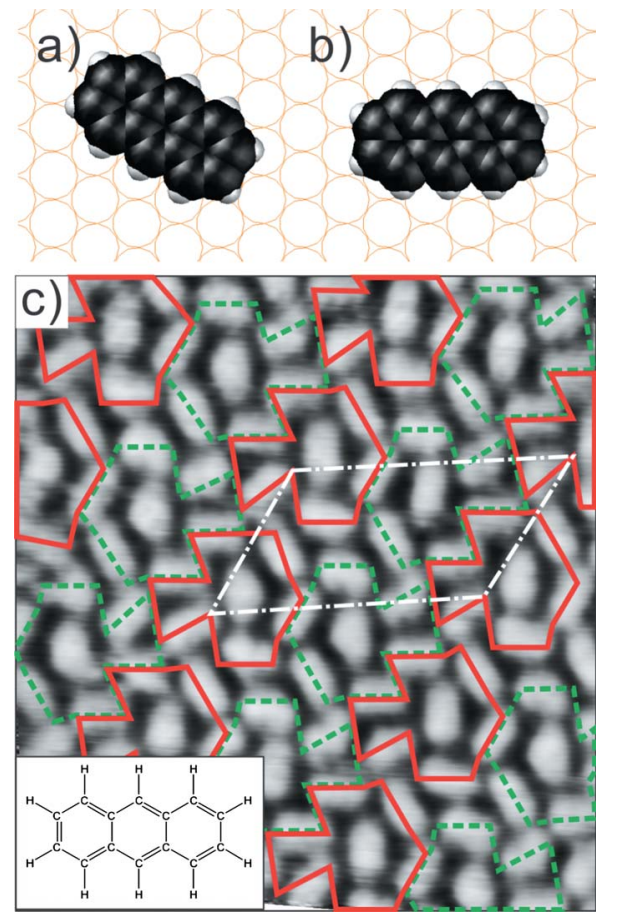

FIG. 1. (Color online) Adsorption configuration of anthracene on $\mathrm{Cu}(111)$ (a) at a hollow site and (b) at a bridge site. Each adsorption configuration is threefold rotational degenerate. Panel (c) shows an STM image of an anthracene superstructure. The unit cell is indicated as a white dotted parallelogram. The superstructure can be described by a geometric motif resembling a fish and its reflection (red solid polylines and green dotted polylines). The inset shows a schematic of anthracene. Imaging parameters: current $=20 \mathrm{pA}$, bias $=-3.1 \mathrm{~V}$, and size $=90 \times 100 \AA^{2}$. 


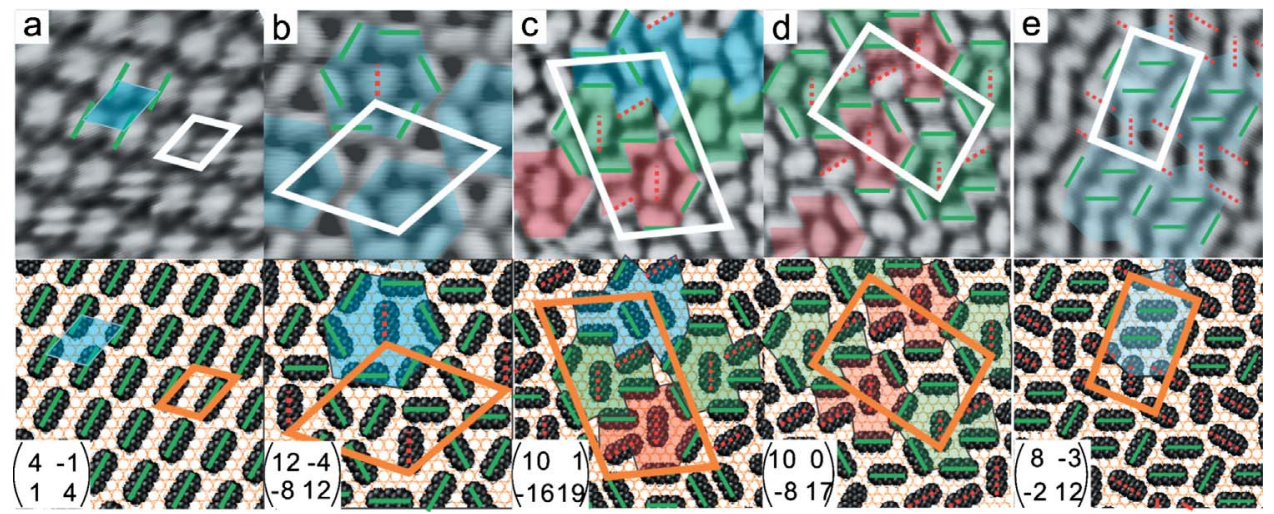

FIG. 2. (Color online) STM images and scaled models of anthracene coverages on $\mathrm{Cu}(111)$. The unit cells are indicated by white lines and structural motifs are highlighted in color shape. Molecules adsorbed at hollow and bridge sites are labeled with green solid bars and red dotted bars, respectively. Panels [(a) and (b)] $54 \AA \times 54 \AA$, panels [(c)-(e)] $61 \AA \times 61 \AA$, Image parameters: (a) current=18 pA, bias $=-2.1 \mathrm{~V}$, (b) current $=19 \mathrm{pA}$, bias $=-2.6 \mathrm{~V}$, (c) current $=20 \mathrm{pA}$, bias $=-3.1 \mathrm{~V}$, (d) current $=20 \mathrm{pA}$, bias $=-3.1 \mathrm{~V}$, and (e) current $=11 \mathrm{pA}$, bias $=-1.6 \mathrm{~V}$.

energy $\sim 0.02 \mathrm{eV}$, i.e., less than anticipated from linear increase with the number of aromatic rings suggested previously. ${ }^{21,22}$

In this study we address anthracene coverages on $\mathrm{Cu}(111)$ that are complete: no bare surface areas remain. We find a large range of adsorbate superstructures which distinguish themselves in geometry, fraction of molecules adsorbed at hollow vs bridge sites and total coverage. In particular, our measurements show that higher coverage leads to an increasing fraction of the molecules in less-favorable adsites. We show that this reduction in average adsorption energy with lateral compression can be interpreted as elastic behavior of the monomolecular film.

All measurements were performed in a low-temperature scanning tunneling microscopy (STM) chamber built on site. Sample preparation involved the typical cycles of sputtering and annealing followed by cooling of the bare surface to $\approx 80 \mathrm{~K}$. Deposition of anthracene through a leak valve proceeded in three incremental steps, each time dosing for $\sim 1$ min at $2 \times 10^{-9}$ torr (an order of magnitude above the chamber base pressure) and imaging to check the coverage. All coverages shown coexist on the resultant surface. Modeling of the adsorption of anthracene involves a nonselfconsistent approach in which the RPBE functional ${ }^{23}$ was used self-consistently to obtain electron densities, which in turn were used to evaluate the nonlocal correlation energies through a first-principles DFT treatment for $\mathrm{vdW}$ interactions. ${ }^{20}$ All calculations use the projector augmented wave method $^{24}$ implemented in the grid-based real-space code GPAW (Ref. 25) and were corroborated using vdW-DF enabled DACAPO. ${ }^{26}$ The supercell was a $5 \times 5 \mathrm{Cu}(111)$ slab of five layers topped by $21 \AA$ A of vacuum, with an anthracene molecule placed parallel to the $\mathrm{Cu}(111)$ surface. The Brillouin zone was sampled only at the $\Gamma$ point. Wave functions, electron densities, and potentials were represented on a $0.16 \AA$ real-space grid. We calculated the adsorption of anthracene at a hollow and a bridge site, to find (besides the mentioned difference in energy) equilibrium heights of the molecule's carbon atoms above the top-layer substrate atoms: $3.49 \AA$ and. $3.52 \AA$, respectively, a small difference, in good agreement with data obtained on benzene (see Ref. 18 and references therein) but in disagreement with the x-ray standing-wave measurements on pentacene/Cu(111) of Ref. 27. In addition to the adsorption geometries discussed above and shown in Figs. 1(a) and 1(b), we investigated a set of alternate configurations which, however, turned out to have prohibitively high energies.

Despite the relative simplicity of the adsorption system, we find highly polymorphic patterns, many of which are characterized by large and complex unit cells. Figure 1(c) shows as an example a $\left(\begin{array}{cc}10 & 0 \\ -8 & 17\end{array}\right)$ superstructure with a unit-cell area of $957 \AA^{2}$, corresponding to 170 substrate atoms.

Given 11 molecules per unit cell, not every molecule in the unit cell interacts directly with every other; consequently, it may be useful to describe the unit cells in terms of the underlying geometric motif that contains the intermolecular geometry. Figure 1(c) highlights how in this superstructure six molecules surround a seventh one in a shape that resembles a "fish." Both the central molecules and one of the "fin" molecules adsorb at less favorable bridge sites, with the remaining molecules adsorbing at the optimal hollow sites. The unit cell consist of a fish shape and an "antifish" shape (with a few shared molecules) connected by a glidereflection line. In total we can identify five different surface patterns, all of which can be found on the same sample in the same preparation.

The $\left(\begin{array}{cc}4 & -1 \\ 1 & 4\end{array}\right)$ superstructure, shown in Fig. 2(a), is the least dense one observed; its unit cell contains only one molecule, which is adsorbed at a hollow site. All molecules are aligned in parallel and are adsorbed at equivalent adsites. There are 17 substrate atoms in the unit cell, giving a coverage of $1 / 17 \mathrm{ML}=0.059 \mathrm{ML}$ with the monolayer $(\mathrm{ML})$ referred to the substrate atom density.

Figure 2(b) shows a $\left(\begin{array}{cc}8 & -3 \\ -2 & 12\end{array}\right)$ superstructure with a unit-cell size of $631 \AA^{2}$, in which six molecules form a symmetric hexagon surrounding a seventh; similar to the structures of Figs. 1(c), 2(c), and 2(d), the surrounded molecule is adsorbed at a bridge site. The density of this film is 16 substrate atoms per molecule, corresponding to coverage of 0.0625 ML. 

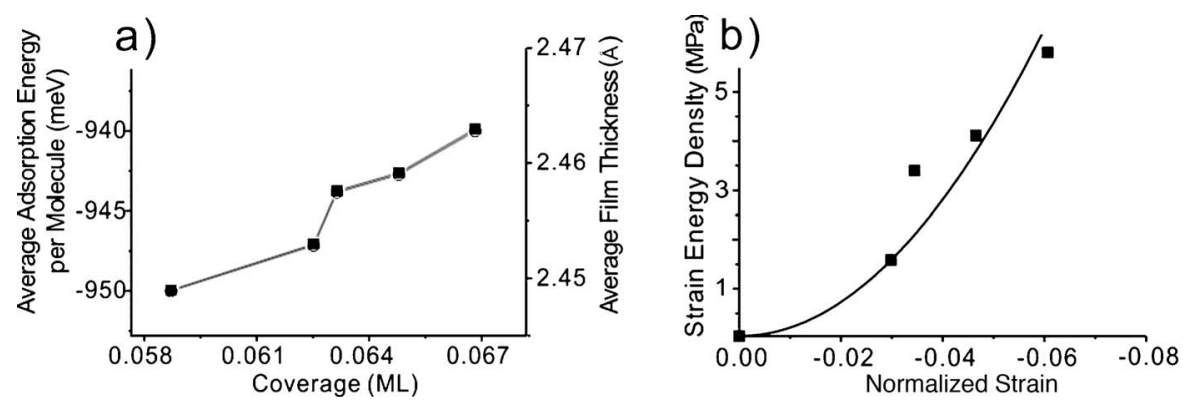

FIG. 3. (a) Variation in the average adsorption energy per molecule and of the average thickness of the anthracene monolayer with coverage; (b) strain energy density obtained from panel (a) as a function of the compressive strain. The quadratic fit yields a Young's modulus of $1.5 \mathrm{GPa}$.
The $\left(\begin{array}{cc}10 & 1 \\ -16 & 19\end{array}\right)$ superstructure of Fig. 2(c) consists of rows of the fish motif of Fig. 1(c) arranged in head-to-tail fashion; between these rows two parallel molecules are found adsorbed at the optimal hollow site. At a size of $1159.9 \AA^{2}$ the unit cell comprises 13 molecules, of which nine come to rest on optimal hollow adsites. The coverage is $0.0631 \mathrm{ML}$.

Figure 2(d) repeats the superstructure of Fig. 1(c); it corresponds to coverage of 0.065 ML. Finally Fig. 2(e) shows a $\left(\begin{array}{cc}8 & -2 \\ -3 & 12\end{array}\right)$ superstructure with a unit-cell size of $507 \AA^{2}$ that features alternating bands of molecules equally distributed between hollow and bridge sites. There are six molecules in each unit cell for a total coverage of $0.067 \mathrm{ML}$, the densest coverage observed.

In total, we observed five different phases that span a compression of the surface area per molecule by more than $10 \%$. The broad geometric diversity of the surface patterns may appear astonishing; however, similar observations have been made in the study of the statistical mechanics of granular media, even as conventional ones as Basmati rice spread out on a vibrating plate. ${ }^{28}$ Bates and Frenkel showed that if spherocylindrical particles with an aspect ratios not exceeding 1:5 are confined in plane, then phases with a variety of local orders exist (at larger aspect ratios, a nematic phase becomes favored). ${ }^{29}$ In contrast to the granular systems of Ref. 28, however, we observe that our patterns always fill the entire space available, i.e., that they correspond to maximum expansion of the system and, thus, its equilibrium at the given confinement. The absence of coexistence of dense with less dense patterns suggests a driving force for expansion of the film, at least up to the most open coverage we observe [Fig. 2(a)], corresponding to optimal adsorption configuration of all anthracene molecules. This invites the questions: (a) what is the character of this restoring force? and (b) can it be understood in terms of continuum elastic theory?

In the different superstructures a varying fraction (0-50\%) of molecules occupy the energetically less-favorable bridge adsorption site. Figure 3(a) plots the average adsorption energy per molecule as a function of coverage, revealing a monotonic relationship between the two. Based on the different anthracene adsorption heights at the bridge and hollow sites, we calculate the average film thickness (subtracting $\frac{1}{2}$ of the copper interlayer spacing), which scales linearly with average adsorption energy [Fig. 3(a)] and increases with compression. Thus, conceptually we find that as the film is compressed from the coverage of Fig. 2(a) (in which each molecule attains its optimal adsorption position), its total energy rises and its thickness increases.

In the study of the mechanical properties of materials, it is common to plot the strain energy density (i.e., the total deformation energy per unit volume) as a function of the strain. Figure 3(b) replots the data set of Fig. 3(a) in this fashion. For an isotropic material subjected to uniform 2D strain in one plane, the stress-strain relationship can be expressed as follows: ${ }^{31}$

$$
\sigma_{i j}=\frac{E}{1+\nu}\left(\varepsilon_{i j}+\frac{\nu}{1-2 \nu} \delta_{i j} \sum_{k} \varepsilon_{k k}\right)
$$

in which $E$ is the Young's modulus, $\sigma$ the stress, $\varepsilon$ the strain, $\nu$ the Poisson's ratio, $\delta$ the Kronecker delta, and $i, j$, and $k$ denote the three spatial directions. For simplicity we regard the anthracene films as isotropic $\left(\varepsilon_{z z}=-\nu \varepsilon_{x x}, \quad \varepsilon_{x x}=\varepsilon_{y y}\right.$ $\left.\rightarrow \varepsilon, \quad \varepsilon_{x y}=\varepsilon_{x z}=\varepsilon_{y z}=0\right)$. Based on the vertical expansion of the film shown in Fig. 3(a), we calculate $\nu=0.095 \pm 0.006$ for all but the least compressed films. For a material with a Hookean response, the strain energy density $U$, i.e., the compression work per unit volume, is $U=(1 / 2) \sum_{i} \sigma_{i i} \varepsilon_{i i}$; for the particular case of an isotropic medium subjected to compressive stress in one plane yet free to expand normal to the plane, $U$ simplifies to

$$
U=\frac{E\left(1-\nu-1 / 2 \nu^{2}\right)}{1-2 \nu} \varepsilon^{2} \approx \frac{E(1-\nu)}{1-2 \nu} \varepsilon^{2}
$$

That is, the strain energy increases quadratically with the strain. A quadratic fit of the data set of Fig. 3(b) yields a value of the Young's modulus of the molecular monolayer of 1.5 GPa, which is substantially less rigid than anthracene crystals $(8.4 \mathrm{GPa})$ or copper $(124 \mathrm{GPa}){ }^{30}$

A combination of Young's modulus and Poisson ratio completely describes the elastic properties of a homogeneous isotropic material. How can we interpret the values obtained? Low Poisson ratios, like the one observed here, are commonly found in porous materials where lateral compression is mainly accommodated by reduction in pore volume rather than requiring transverse expansion of the object. If a porous material is deformed, a relatively small amount of matter has to deform compared to the overall volume of the object, and a relatively low Young's modulus results. This is also found here, suggesting that the closer packing of the molecules observed in our STM experiments can indeed be interpreted as compression of a porous material, revealing a remarkable congruence of macroscopic and microscopic behavior. It should be pointed out that we have treated the anthracene molecules as hard, noninteracting particles; the interactions evaluated here stem exclusively from the variation in the vdW interaction of anthracene across the substrate. 
In summary, we have investigated the adsorption geometry of anthracene on $\mathrm{Cu}(111)$ and found-despite the adsorption system's comparative simplicity-a large number of highly ordered superstructures with coverage varying by $>10 \%$. The polymorphic nature of the anthracene film coincides with the behavior of macroscopic granular media of similar aspect ratio. Modeling of the adsorption behavior of the system in first-principles DFT with inclusion of vdW interactions provides us parameters which we use further to evaluate properties of the superstructures in terms of continuum elastic theory. Based exclusively on the lateral variation of the $\mathrm{vdW}$ interactions between the adsorbates and the substrate, the monolayer turns out to have $\sim 20 \%$ of the Young's modulus of the molecular bulk and a Poisson ratio of $\sim 0.1$.

We gratefully acknowledge joint NSF under Grants No. CHE 07-50334 (T.L.E) and No. CHE 07-49949 (L.B.), a joint DOE under Grant No. DE-FG02-07ER15842 (T.S.R. and L.B.), and support from the Swedish Research Council (P.H.). T.L.E. acknowledges secondary support from NSFMRSEC under Grant No. DMR 05-20471 and ancillary support from CNAM.

\footnotetext{
*ludwig.bartels@ucr.edu

${ }^{1}$ C. Jaeger, Rock Mechanics and Engineering (Cambridge University Press, Cambridge, England, New York, 1979).

${ }^{2}$ Y. Fujita, N. A. Duncan, and J. C. Lotz, J. Orthop. Res. 15, 814 (1997).

${ }^{3}$ M. Weber, R. Koch, and K. H. Rieder, Phys. Rev. Lett. 73, 1166 (1994).

${ }^{4}$ H. Ibach, Surf. Sci. Rep. 29, 195 (1997).

${ }^{5}$ P. Müller and A. Saul, Surf. Sci. Rep. 54, 157 (2004).

${ }^{6}$ L. Bartels, Nat. Chem. 2, 87 (2010).

${ }^{7}$ S. De Feyter and F. C. De Schryver, Chem. Soc. Rev. 32, 139 (2003).

${ }^{8}$ G. Pawin, K. L. Wong, K. Y. Kwon, and L. Bartels, Science 313, 961 (2006).

${ }^{9}$ J. A. Theobald, N. S. Oxtoby, M. A. Phillips, N. R. Champness, and P. H. Beton, Nature (London) 424, 1029 (2003).

${ }^{10}$ U. Schlickum et al., Nano Lett. 7, 3813 (2007).

${ }^{11}$ C. B. France, P. G. Schroeder, J. C. Forsythe, and B. A. Parkinson, Langmuir 19, 1274 (2003).

${ }^{12}$ S. Lukas, G. Witte, and C. Wöll, Phys. Rev. Lett. 88, 028301 (2001).

${ }^{13}$ L. J. Wan and K. Itaya, Langmuir 13, 7173 (1997).

${ }^{14}$ G. Witte and C. Wöll, J. Mater. Res. 19, 1889 (2004).

${ }^{15}$ F. Ample and C. Joachim, Surf. Sci. 600, 3243 (2006).

${ }^{16}$ S. Lukas, S. Vollmer, G. Witte, and C. Wöll, J. Chem. Phys. 114, 10123 (2001).

${ }^{17}$ E. Mete, I. Demiroğlu, M. F. Danişman, and Ş. Ellialtioğlu, J. Phys. Chem. C 114, 2724 (2010).
}

${ }^{18}$ K. Berland, T. L. Einstein, and P. Hyldgaard, Phys. Rev. B 80, 155431 (2009).

${ }^{19}$ A. Bilić, J. R. Reimers, N. S. Hush, R. C. Hoft, and M. J. Ford, J. Chem. Theory Comput. 2, 1093 (2006).

${ }^{20}$ M. Dion, H. Rydberg, E. Schröder, D. C. Langreth, and B. I. Lundqvist, Phys. Rev. Lett. 92, 246401 (2004).

${ }^{21}$ S. D. Chakarova-Käck, E. Schröder, B. I. Lundqvist, and D. C. Langreth, Phys. Rev. Lett. 96, 146107 (2006).

${ }^{22}$ S. D. Chakarova-Käck, A. Vojvodic, J. Kleis, P. Hyldgaard, and E. Schröder, New J. Phys. 12, 013017 (2010).

${ }^{23}$ B. Hammer, L. B. Hansen, and J. K. Nørskov, Phys. Rev. B 59, 7413 (1999).

${ }^{24}$ P. E. Blöchl, O. Jepsen, and O. K. Andersen, Phys. Rev. B 49, 16223 (1994).

${ }^{25}$ J. J. Mortensen, L. B. Hansen, and K. W. Jacobsen, Phys. Rev. B 71, 035109 (2005).

${ }^{26}$ D. C. Langreth, M. Dion, H. Rydberg, E. Schröder, P. Hyldgaard, and B. I. Lundqvist, Int. J. Quantum Chem. 101, 599 (2005).

${ }^{27}$ N. Koch et al., J. Am. Chem. Soc. 130, 7300 (2008).

${ }^{28}$ V. Narayan, N. Menon, and S. Ramaswamy, J. Stat. Mech.: Theory Exp. 2006, P01005.

${ }^{29}$ M. A. Bates and D. Frenkel, J. Chem. Phys. 112, 10034 (2000).

${ }^{30} \mathrm{G}$. Simmons and H. Wang, Single Crystal Elastic Constants and Calculated Aggregate Properties: A Handbook (MIT Press, Cambridge, Massachusetts, 1971).

${ }^{31}$ L. D. Landau and E. M. Lifshitz, Theory of Elasticity (Pergamon, Oxford, 1970), p. 14. 